Molecules 2001, 6, M219

\title{
2-Bromo-3-ethylthiazolium Tetrafluoroborate (BET)
}

\section{Györgyi Kovács $^{1 *}$, Zoltán Kele ${ }^{1}$, Péter Forgó ${ }^{2}$ and Lajos Kovács ${ }^{1}$}

1. Department of Medicinal Chemistry, University of Szeged, Dóm tér 8, H-6720 Szeged, Hungary. Phone: +36 625451 45, Fax: +36 625459 71, *E-mail: gyorgyi@ovrisc.mdche.u-szeged.hu 2. Department of Organic Chemistry, University of Szeged, Dóm tér 8, H-6720 Szeged, Hungary

Received: 20 January 2001 / Accepted: 15 May 2001 / Published: 25 May 2001
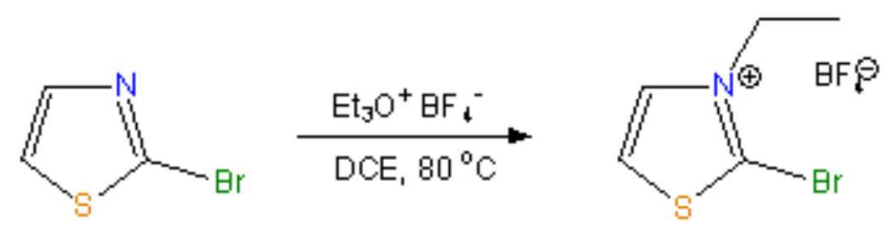

Recent progress in peptide synthesis resulted in the elaboration of novel coupling agents. One successful approach involves the application of thiazolium salts, e.g. 2-bromo-3-ethyl-4-methylthiazolium tetrafluoroborate (BEMT) [1-3]. This compound can be prepared from 2-bromo-3-ethyl-4-methylthiazole $[1,2]$. We have found that a simpler analogue, 2-bromo-3-ethylthiazolium tetrafluoroborate (BET), can be prepared in convenient steps from the commercially available 2-aminothiazole using the procedure by Dondoni et al. [4] to obtain 2-bromothiazole which, in turn, was readily transformed into the title compound. In our experience BET is a highly efficient coupling reagent in the synthesis of peptide nucleic acid (PNA) oligomers in solution phase.

To a stirred solution of 2-bromothiazole [4] (1.78 mL, $20.0 \mathrm{mmol})$ in 1,2-dichloroethane (DCE, $20 \mathrm{~mL})$ the solution of triethyloxonium tetrafluoroborate $(11.4 \mathrm{~g}, 60.0 \mathrm{mmol})$ in $\mathrm{DCE}(60 \mathrm{~mL})$ was added over 45 min. at $80^{\circ} \mathrm{C}$. When the reaction was complete $(16 \mathrm{~h})$ according to TLC (n-buthanol : acetic acid : water $=4: 1: 1)$ the solution was concentrated in vacuo. After the residue was precipitated from diethyl ether it was recrystallized from $a b s$. acetonitrile/ethyl acetate to afford white plates. Yield: $4.48 \mathrm{~g}(80 \%)$.

Mp: $137.9-139.2^{\circ} \mathrm{C}$.

${ }^{1} \mathrm{H}$ NMR (DMSO-d $\left.6,500 \mathrm{MHz}, \mathrm{d}, \mathrm{ppm}\right): 1.47$ (t, $J=7.0 \mathrm{~Hz}, 3 \mathrm{H}, \mathrm{CH}_{2} \mathrm{CH}_{3}$ ); 4.52 (q, $J=7.0 \mathrm{~Hz}, 2 \mathrm{H}$, $\left.\mathrm{CH}_{2} \mathrm{CH}_{3}\right) ; 8.35(\mathrm{~d}, J=4.1 \mathrm{~Hz}, 1 \mathrm{H}$, aryl); $8.56(\mathrm{~d}, J=4.1 \mathrm{~Hz}, 1 \mathrm{H}$, aryl).

${ }^{13} \mathrm{C}$ NMR (DMSO-d6, $\left.125 \mathrm{MHz}, \mathrm{d}, \mathrm{ppm}\right): 14.19\left(\mathrm{CH}_{2} \mathrm{CH}_{3}\right) ; 50.61\left(\mathrm{CH}_{2} \mathrm{CH}_{3}\right) ; 127.45(\mathrm{C}-5) ; 137.73$ (C-4); 146.50 (C-2).

ESI-MS (m/z, \%): $191.8\left(100,\left[\mathrm{C}_{5} \mathrm{H}_{7}{ }^{79} \mathrm{BrNS}^{+}\right) ; 193.8\left(97,\left[\mathrm{C}_{5} \mathrm{H}_{7}{ }^{81} \mathrm{BrNS}^{+}\right)\right.\right.$.

Anal. cald. for $\mathrm{C}_{5} \mathrm{H}_{7} \mathrm{BBrF}_{4} \mathrm{NS}$ (279.891): C, 21.45; H, 2.52; Br, 28.55; F, 27.15; N, 5.00; S, 11.46; found C, 21.47; H, 2.51; Br, 28.52; F, 27.20; N, 5.01; S, 11.44.

\section{References}

1. Li, P.; Xu, J. C. Tetrahedron Lett.1999, 40, 8301-8304.

2. Li, P.; Xu, J. C. J. Org. Chem. 2000, 65, 2951-2958.

3. Li, P.; Xu, J. C. Tetrahedron 2000, 56, 8119-8131.

4. Dondoni, A. in Modern synthetic methods; Scheffold, R., Ed.; Verlag Helvetica Chimica Acta \& Verlag Chemie: Basel, Weinheim, New York, Cambridge, 1992; Vol. 6, p 385.

Sample availability: sample available from the authors and MDPI. 
(C) 2001 MDPI, Basel, Switzerland. All rights reserved. Molecules website www.mdpi.org/molecules/

\title{
n
}

\author{
(1)
}

\section{(1)} (1) (1) (1) . . . . . . . . . . . . . . . .

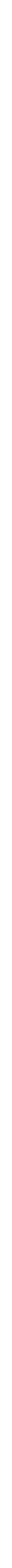

